\title{
Physical and chemical variations within the W3 star-forming region
}

\section{I. $\mathrm{SO}_{2}, \mathrm{CH}_{3} \mathrm{OH}$ and $\mathrm{H}_{2} \mathrm{CO}$}

\author{
F.P. Helmich ${ }^{1}$, D.J. Jansen ${ }^{1}$, Th. de Graauw ${ }^{2}$, T.D. Groesbeck ${ }^{3}$, and E.F. van Dishoeck ${ }^{1}$ \\ 1 Leiden Observatory, P.O.-Box 9513, 2300 RA Leiden, The Netherlands \\ 2 SRON-Groningen, P.O.-Box 800, 9700 AV Groningen, The Netherlands \\ ${ }^{3}$ Division of Physics, Mathematics \& Astronomy, California Institute of Technology, Pasadena, CA 91125, USA
}

Received 5 July 1993 / Accepted 11 September 1993

\begin{abstract}
Submillimeter observations of $\mathrm{SO}_{2}, \mathrm{CH}_{3} \mathrm{OH}$ and $\mathrm{H}_{2} \mathrm{CO}$ towards three positions in the W3 star-forming molecular cloud (IRS4, IRS5 and W3 $\left(\mathrm{H}_{2} \mathrm{O}\right)$ ) are presented. Significant differences in the physical and chemical conditions between the three sources are found. The gas temperature appears highest with $T \approx 220 \mathrm{~K}$ towards $\mathrm{W} 3\left(\mathrm{H}_{2} \mathrm{O}\right)$, followed by $T \approx 100 \mathrm{~K}$ towards IRS5 and $T \approx 55 \mathrm{~K}$ towards IRS4. Densities are $10^{6} \mathrm{~cm}^{-3}$ or larger. The chemical composition of W3 $\left(\mathrm{H}_{2} \mathrm{O}\right)$ with strong $\mathrm{CH}_{3} \mathrm{OH}$ lines resembles that of a "hot core" type region. In contrast, W3 IRS5 appears at an earlier evolutionary stage where complex molecules are still depleted onto grains, but where energetic events have produced strong $\mathrm{SO}_{2}$ emission. W3 IRS4 is the most evolved region with a chemistry similar to that found in quiescent molecular clouds. Here the time scale since star formation has probably been long enough for the gas-phase ion-molecule chemistry to become dominant again.
\end{abstract}

Key words: ISM: molecules - ISM: clouds - ISM: individual: W3 IRS5, W3 IRS4, W3 $\left(\mathrm{H}_{2} \mathrm{O}\right)$

\section{Introduction}

The W3 region consists of a giant molecular cloud with numerous $\mathrm{H}$ II regions and luminous infrared sources. In its core, millimeter continuum observations (Richardson et al. 1989) have shown the existence of two dense clumps of about $2000 M_{\odot}$ associated with the luminous infrared sources IRS4 and IRS5 (Wynn-Williams et al. 1972). Both sources have a total luminosity of $(1-2) \cdot 10^{5} L_{\odot}$, and are separated by about $1 .{ }^{\prime} 14$, corresponding to $0.83 \mathrm{pc}$ at the distance of the complex of $2.3 \mathrm{kpc}$ (Georgelin \& Georgelin 1976). Of comparable luminosity and

Send offprint requests to: F.P. Helmich mass is $\mathrm{W} 3\left(\mathrm{H}_{2} \mathrm{O}\right)$ located at the place of the water masers only $7^{\prime \prime}$ from the well-known compact $\mathrm{H}$ II region W3(OH). W3(OH) is associated with many $\mathrm{OH}$ masers and can be found about $16^{\prime}$ (11.2 pc) SE of the core (Dickel et al. 1980). Thus, the W3 cloud contains several sites of high-mass star formation which appear to be at different evolutionary stages.

In order to investigate their physical and chemical state, we are in the process of obtaining an unbiased spectral survey of these three sources in the $345 \mathrm{GHz}$ atmospheric window and selected parts of the $230 \mathrm{GHz}$ window with the 15-m James Clerk Maxwell Telescope ${ }^{1}$ (JCMT) on Mauna Kea, Hawaii. The main advantage of submillimeter observations compared with earlier millimeter data (e.g. Dickel et al. 1980; Hayashi et al. 1989; Wilson et al. 1991) is that the higher frequency observations probe primarily the warm, dense gas associated with the young stellar objects rather than the cold surrounding cloud. Also, they have sufficiently high spatial resolution to separate the IRS4 and IRS5 regions. Moreover, both infrared sources are bright enough at near-infrared wavelengths to permit absorption line studies of both the gas and the dust composition in front of the source, thus providing important complementary information (Mitchell et al. 1990; Tielens et al. 1991; Allamandola et al. 1992). We present here the initial results of the submillimeter study.

\section{Observations and results}

The observations were performed in January, August and December 1992 with the JCMT. As the frontends, we used the facility heterodyne double-side band receivers. At $345 \mathrm{GHz}$, the SIS receiver $\mathrm{B} 3 \mathrm{i}$ was employed during all three observing sessions, whereas at $230 \mathrm{GHz}$, the Schottky receiver A1 was

\footnotetext{
The James Clerk Maxwell Telescope is operated by the Royal Observatory Edinburgh on behalf of the Science and Engineering Research Council of the United Kingdom, the Netherlands Organisation for Scientific Research and the National Research Council of Canada.
} 
Table 1. Receiver characteristics

\begin{tabular}{lcccc}
\hline Rec. & $\eta_{\mathrm{MB}}$ & $\begin{array}{c}T_{\mathrm{rx}} \\
(\mathrm{K})\end{array}$ & $\begin{array}{c}T_{\text {sys }} \\
(\mathrm{K})\end{array}$ & $\begin{array}{c}\text { Beam } \\
(\operatorname{arcsec})\end{array}$ \\
\hline A1 & 0.7 (Jan.) & 350 & $\sim 850$ & 21 \\
A2 & 0.5 (Aug.)/0.63(Dec.) & $\sim 100$ & $\sim 500-700$ & 21 \\
B3i & 0.6 (Jan.)/0.53(Dec.) & 200 & $\sim 600-1000$ & 15 \\
\hline
\end{tabular}

From Matthews (1991) and Baas (1992)

available in January, and the SIS receiver A2 in August and December. For A 2 and $\mathrm{B} 3 \mathrm{i}$, the local oscillator frequency is such that the upper and lower side bands are $3 \mathrm{GHz}$ apart while for A1 this difference is $7.88 \mathrm{GHz}$. No systematic side band check of the lines was performed, since in most cases the line crowding is such that there is little ambiguity in the identification. The 2048 channel acousto-optical spectrometer AOSC was used as the backend in all observations. It has a bandwidth of $500 \mathrm{MHz}$ and a channel spacing of $250 \mathrm{kHz}$, with an effective resolution of 2 channels corresponding to 0.65 and $0.43 \mathrm{~km} \mathrm{~s}^{-1}$ at 230 and $345 \mathrm{GHz}$, respectively. The observing conditions were good in January and December, but poor in August when no $345 \mathrm{GHz}$ data were obtained at all. Integration times of $30 \mathrm{~min}-$ utes on+off source resulted in typical $1 \sigma$ noise levels in $T_{\mathrm{A}}^{*}$ of 30 (A2)-50 (B3i) $\mathrm{mK}$ per resolution element ( 2 channels).

Calibration was performed according to Kutner \& Ulich (1981) using a single channel total power detector covering the entire bandwidth of the receiver. However, due to a mismatch in the mixer the data obtained with receiver B3i showed a significant dip in the response of the AOS over a specific range of channels. This dip was calibrated to be about $30 \%$ of the continuum from observations of a continuum source such as a planet, and this channel-to-channel variation was removed quite effectively using a "flat field" supplied by the JCMT staff. No indications were found for a dependence of this "flat field" on upper or lower side band. The adopted main-beam efficiencies $\eta_{\mathrm{MB}}$ have been obtained from measurements of planets and are summarized in Table 1. The absolute uncertainties in the resulting temperatures $T_{\mathrm{MB}}$ are estimated to be about $30 \%$ from repeated observations and comparison with other telescopes. The relative uncertainties can be significantly less. The beam size of the JCMT is about $21^{\prime \prime}$ at $230 \mathrm{GHz}$, decreasing to $15^{\prime \prime}$ at $345 \mathrm{GHz}$. The observations in January were performed in position-switch mode with large offsets of $15^{\prime}-60^{\prime}$ in right ascension. Since the baselines of these spectra suffered from severe ripples with widths comparable to those of the lines in W3, beam-switching by means of a wobbling secondary was used in August and December with a $+180^{\prime \prime}$ throw in azimuth and a frequency of $1 \mathrm{~Hz}$. Test observations showed at most a $10 \%$ reduction in the $\mathrm{C}^{18} \mathrm{O}$ 2-1 intensity in this observing mode compared with the large position switch, and even smaller differences for high excitation lines of rarer molecules such as those discussed in this paper. Pointing was checked regularly on the continuum of $\mathrm{W} 3(\mathrm{OH})$ and was found to be consistent within $3^{\prime \prime}$.
The spectra obtained to date cover about $15 \mathrm{GHz}$ in the $345 \mathrm{GHz}$ window, and about $15 \mathrm{GHz}$ at $230 \mathrm{GHz}$. In Figs. 1 and 2 , examples of spectra are presented. The differences between the three sources are immediately apparent. At the W3 IRS4 position, lines of simple molecules such as $\mathrm{CO}, \mathrm{CS}, \mathrm{HCO}^{+}$and $\mathrm{H}_{2} \mathrm{CO}$ are primarily seen. These molecules are also found towards IRS5, but here silicon- and sulfur-bearing molecules such as $\mathrm{SiO}$ and $\mathrm{SO}_{2}$ become prominent as well. By far the most complex spectra are found at the $\mathrm{W} 3\left(\mathrm{H}_{2} \mathrm{O}\right)$ position, where several hundreds of molecular lines have been detected. With a few exceptions these lines can be readily identified from submillimeter catalogs. A complete list will be given in a subsequent paper. Here we concentrate on three molecules: $\mathrm{SO}_{2}, \mathrm{H}_{2} \mathrm{CO}$ and $\mathrm{CH}_{3} \mathrm{OH}$. These molecules are representative of the different types of chemistry and are also excellent diagnostics of the physical parameters. As Figs. 1 and 2 show, they behave very differently in the three sources: $\mathrm{CH}_{3} \mathrm{OH}$ is strong in $\mathrm{W} 3\left(\mathrm{H}_{2} \mathrm{O}\right)$, moderate towards IRS4 and weak towards IRS5, whereas $\mathrm{SO}_{2}$ is strong towards IRS5 and $\mathrm{W} 3\left(\mathrm{H}_{2} \mathrm{O}\right)$, but weak towards IRS4.

Since W3 is a complex region, care has to be taken in the analysis of various possible overlapping components. Towards IRS4, we found all line profiles (except those of ${ }^{12} \mathrm{CO}$ ) to be well represented by single Gaussians with $\Delta V \approx(3.5 \pm 1.5) \mathrm{km} \mathrm{s}^{-1}$ at $V_{\mathrm{LSR}} \approx-44 \mathrm{~km} \mathrm{~s}^{-1}$. Similarly, towards IRS5 all lines except those of $\mathrm{SO}_{2}$ were well fitted with $\Delta V \approx(4.4 \pm 1.5) \mathrm{km} \mathrm{s}^{-1}$ at $V_{\mathrm{LSR}} \approx-39 \mathrm{~km} \mathrm{~s}^{-1}$. The $\mathrm{SO}_{2}$ lines have slightly larger widths of $\Delta V \approx(6.2 \pm 1.5) \mathrm{km} \mathrm{s}^{-1}$ at $-39 \mathrm{~km} \mathrm{~s}^{-1}$. No evidence was found for two overlapping components at $V_{\mathrm{LSR}}=-38$ and $-41 \mathrm{~km} \mathrm{~s}^{-1}$, as inferred by Mitchell et al. (1992) from CO observations. The $\mathrm{W} 3\left(\mathrm{H}_{2} \mathrm{O}\right)$ position is known to contain emission at $V_{\mathrm{LSR}}=-47 \mathrm{~km} \mathrm{~s}^{-1}$ with $\Delta V>4 \mathrm{~km} \mathrm{~s}^{-1}$, as well as a component at $V_{\mathrm{LSR}}=-44.5 \mathrm{~km} \mathrm{~s}^{-1}$ with a much narrower line width characterizing the gas towards $\mathrm{W} 3(\mathrm{OH})$ (Mauersberger et al. 1988). Since the latter source is only $7^{\prime \prime}$ away from $\mathrm{W} 3\left(\mathrm{H}_{2} \mathrm{O}\right)$, some emission from it will be picked up by the 15-20" JCMT beam. However, because the physical and chemical characteristics of $\mathrm{W} 3(\mathrm{OH})$ are quite different from those of $\mathrm{W} 3\left(\mathrm{H}_{2} \mathrm{O}\right)$, surprisingly little contamination was found for the lines towards $\mathrm{W} 3\left(\mathrm{H}_{2} \mathrm{O}\right)$ analyzed in this paper; virtually all of them are well characterized by single Gaussians at $-47 \mathrm{~km} \mathrm{~s}^{-1}$.

\section{Analysis}

\subsection{Rotation diagrams of $\mathrm{SO}_{2}$ and $\mathrm{CH}_{3} \mathrm{OH}$}

In order to constrain the physical conditions, in particular the temperature, we have first constructed rotation diagrams for the three molecules. $\mathrm{SO}_{2}$ and $\mathrm{CH}_{3} \mathrm{OH}$ are especially useful in this respect, because they are asymmetric rotors with many lines throughout the submillimeter region. The measured integrated main-beam temperatures of the lines, $\int T_{\mathrm{MB}} d V\left(\mathrm{~K} \mathrm{~km} \mathrm{~s}^{-1}\right)$, are related to the column densities of the molecules in the upper level $N_{\mathrm{u}}$ by

$N_{\mathrm{u}} / g_{\mathrm{u}}=\frac{N_{\mathrm{tot}}}{Q\left(T_{\mathrm{rot}}\right)} e^{-E_{\mathrm{u}} / T_{\mathrm{rot}}}=\frac{1.67 \cdot 10^{14}}{\nu \mu^{2} S} \int T_{\mathrm{MB}} d V$ 


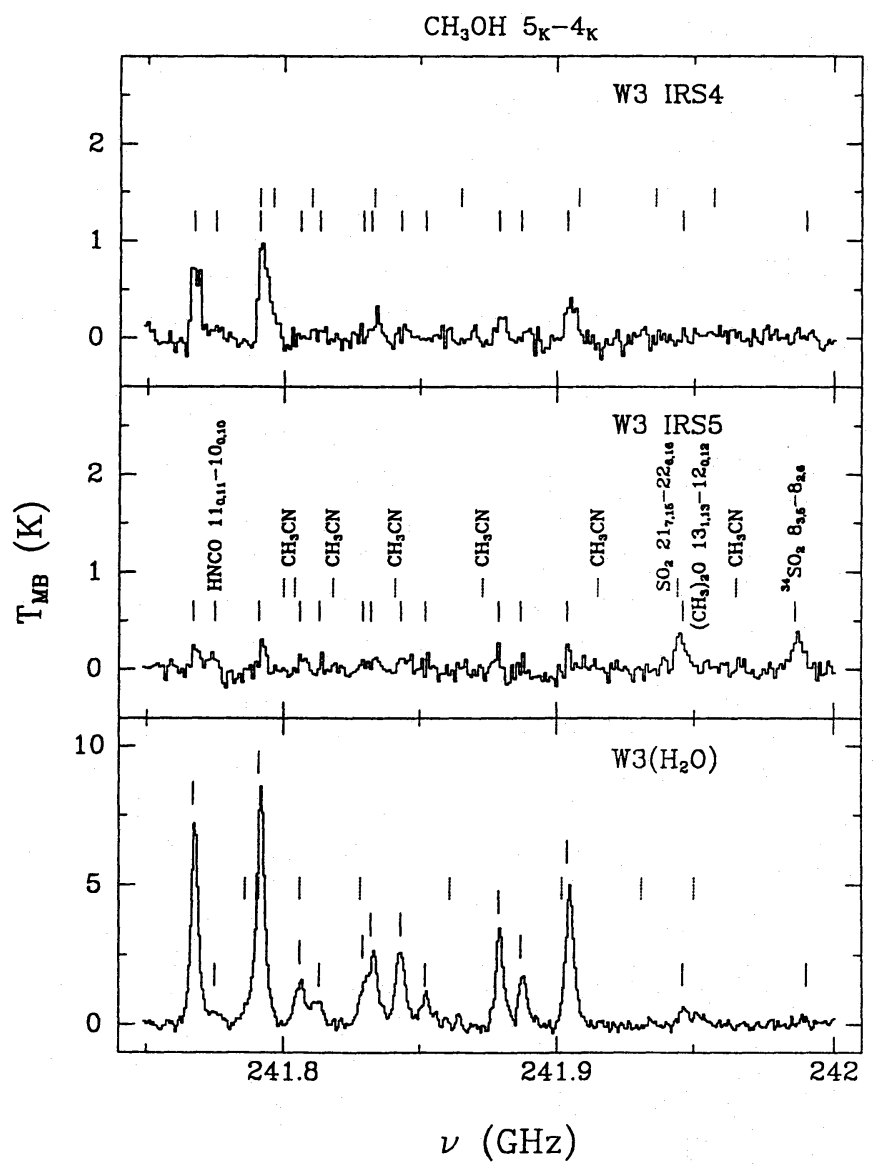

Fig. 1. Spectra of the methanol band at $241.8 \mathrm{GHz}$ towards IRS4 $\left(02^{\mathrm{h}} 21^{\mathrm{m}} 43^{\mathrm{s}} .5+61^{\circ} 52^{\prime} 49^{\prime \prime}, \quad V_{L S R}=-44 \mathrm{~km} \mathrm{~s}^{-1}\right)$, IRS5 $\left(02^{\mathrm{h}} 21^{\mathrm{m}} 53^{\mathrm{s}} .1+61^{\circ} 52^{\prime} 20^{\prime \prime}, \quad V_{L S R}=-39 \mathrm{~km} \mathrm{~s}^{-1}\right)$ and $\mathrm{W} 3\left(\mathrm{H}_{2} \mathrm{O}\right)$ $\left(02^{\mathrm{h}} 23^{\mathrm{m}} 17^{\mathrm{s}} .3+61^{\circ} 38^{\prime} 58^{\prime \prime}, V_{L S R}=-47 \mathrm{~km} \mathrm{~s}^{-1}\right)$. Coordinates are for epoch B1950.0. All unlabeled solid tickmarks in the IRS5 spectrum indicate the positions of methanol lines of the $55_{K}-4_{K}$ band. All lines except for $\mathrm{SO}_{2} 21_{7,15}-22_{6,16}(238993 \mathrm{MHz})$ and the $\mathrm{CH}_{3} \mathrm{CN}$ $13_{K}-12_{K}$ lines $(239138,239133,239119,239096,239064,239022$, and $238972 \mathrm{MHz}$ ) occur in the upper sideband of which the frequency is given as the ordinate. The $\mathrm{SO}_{2}$ and $\mathrm{CH}_{3} \mathrm{CN}$ lines in the lower sideband are labeled with dotted tickmarks. The contribution of $\mathrm{CH}_{3} \mathrm{CN}$ to the methanol band in $\mathrm{W} 3\left(\mathrm{H}_{2} \mathrm{O}\right)$ has been calculated from its unblended $14_{K}-13_{K}$ band at $257 \mathrm{GHz}$. The rest frequency for $\mathrm{HNCO}$ $11_{0,11}-10_{0,10}$ is $241774 \mathrm{MHz}$; for ${ }^{34} \mathrm{SO}_{2} 8_{3,5}-8_{2,6} 241985 \mathrm{MHz}$ and for $\left(\mathrm{CH}_{3}\right)_{2} \mathrm{O} 13_{1,13}-12_{0,12} 241946 \mathrm{MHz}$

where $g_{\mathrm{u}}$ is the statistical weight of level $\mathrm{u} ; N_{\text {tot }}$ is the total column density in $\mathrm{cm}^{-2} ; Q\left(T_{\text {rot }}\right)$ is the partition function for the rotation temperature $T_{\text {rot }} ; E_{\mathrm{u}}$ is the energy of the upper level in Kelvin; $\nu$ is the frequency in $\mathrm{GHz} ; \mu$ is the permanent dipole moment in Debye; and $S$ is the line strength value (see Blake et al. 1987). Thus, a logarithmic plot of the quantity on the right-hand side of Eq. (1) as a function of $E_{\mathrm{u}}$ provides a straight line with slope $1 / T_{\text {rot }}$ and intercept $N_{\text {tot }} / Q\left(T_{\text {rot }}\right)$. This method assumes that all level populations can be characterized by a single excitation temperature $T_{\text {rot }}$ and that the lines are optically thin.

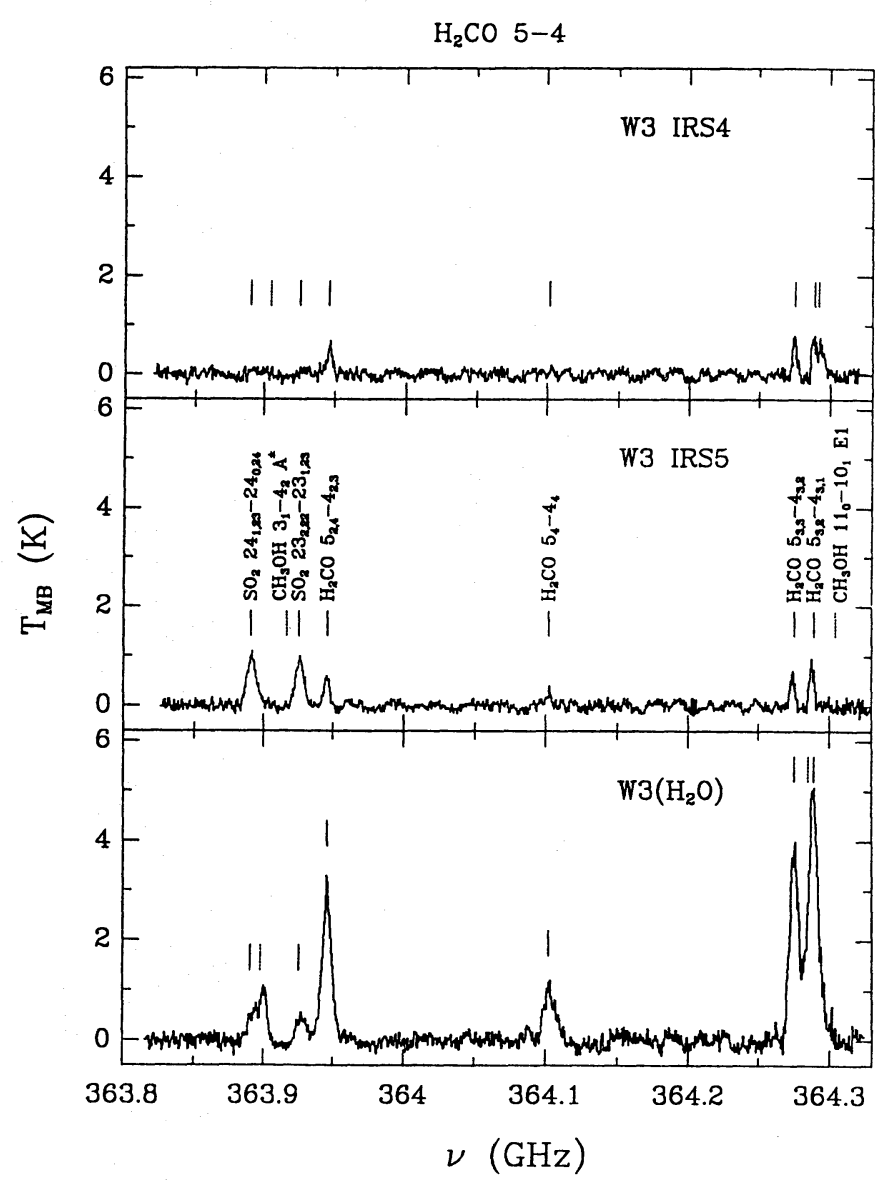

Fig. 2. Spectra containing $\mathrm{H}_{2} \mathrm{CO} 5-4$ lines towards the three sources IRS4, IRS5 and $\mathrm{W} 3\left(\mathrm{H}_{2} \mathrm{O}\right)$. The rest frequency in the upper sideband is given as the ordinate; only the $\mathrm{CH}_{3} \mathrm{OH} 11_{0}-10_{1} E 1(360849 \mathrm{MHz})$ and $\mathrm{CH}_{3} \mathrm{OH} 3_{1}-4_{2} A^{ \pm}$(361236 MHz) lines occur in the lower side band (dotted tickmarks). Note the strong $\mathrm{SO}_{2}$ lines $23_{2,22}-23_{1,23}$ at $363926 \mathrm{MHz}$ and $24_{1,23}-24_{0,24}$ at $363891 \mathrm{MHz}$ in the IRS5 and W3 $\left(\mathrm{H}_{2} \mathrm{O}\right)$ spectra

In Fig. 3, the rotation diagrams for $\mathrm{SO}_{2}$ in the three sources are presented. Towards IRS4, only a few lines have been detected, which can be well fitted with an excitation temperature $T_{\text {rot }} \approx 42 \pm 13 \mathrm{~K}$. Towards IRS5 and $\mathrm{W} 3\left(\mathrm{H}_{2} \mathrm{O}\right)$, the $\mathrm{SO}_{2}$ lines are considerably stronger and more excited: lines originating from energy levels up to $600 \mathrm{~K}$ have been detected. The leastsquares fit through all data points gives excitation temperatures in excess of $150 \mathrm{~K}$ in both cases. The ${ }^{34} \mathrm{SO}_{2}$ data for IRS5 and W3 $\left(\mathrm{H}_{2} \mathrm{O}\right)$ are included in Fig. 3, and even though they do not cover as large an energy range, they still give excitation temperatures in excess of $100 \mathrm{~K}$. Although the rotational temperatures $T_{\text {rot }}$ are not necessarily equal to the gas kinetic temperatures, they strongly suggest that the gas towards IRS5 and W3 $\left(\mathrm{H}_{2} \mathrm{O}\right)$ is warmer than that towards IRS4.

The scatter in the IRS5 and $\mathrm{W} 3\left(\mathrm{H}_{2} \mathrm{O}\right)$ rotation diagrams appears larger than can be explained on the basis of uncertainties in the calibration alone. Unresolved blends could cause the strengths of some of the lines to be overestimated, but would not explain why other observed lines fall below the average. 


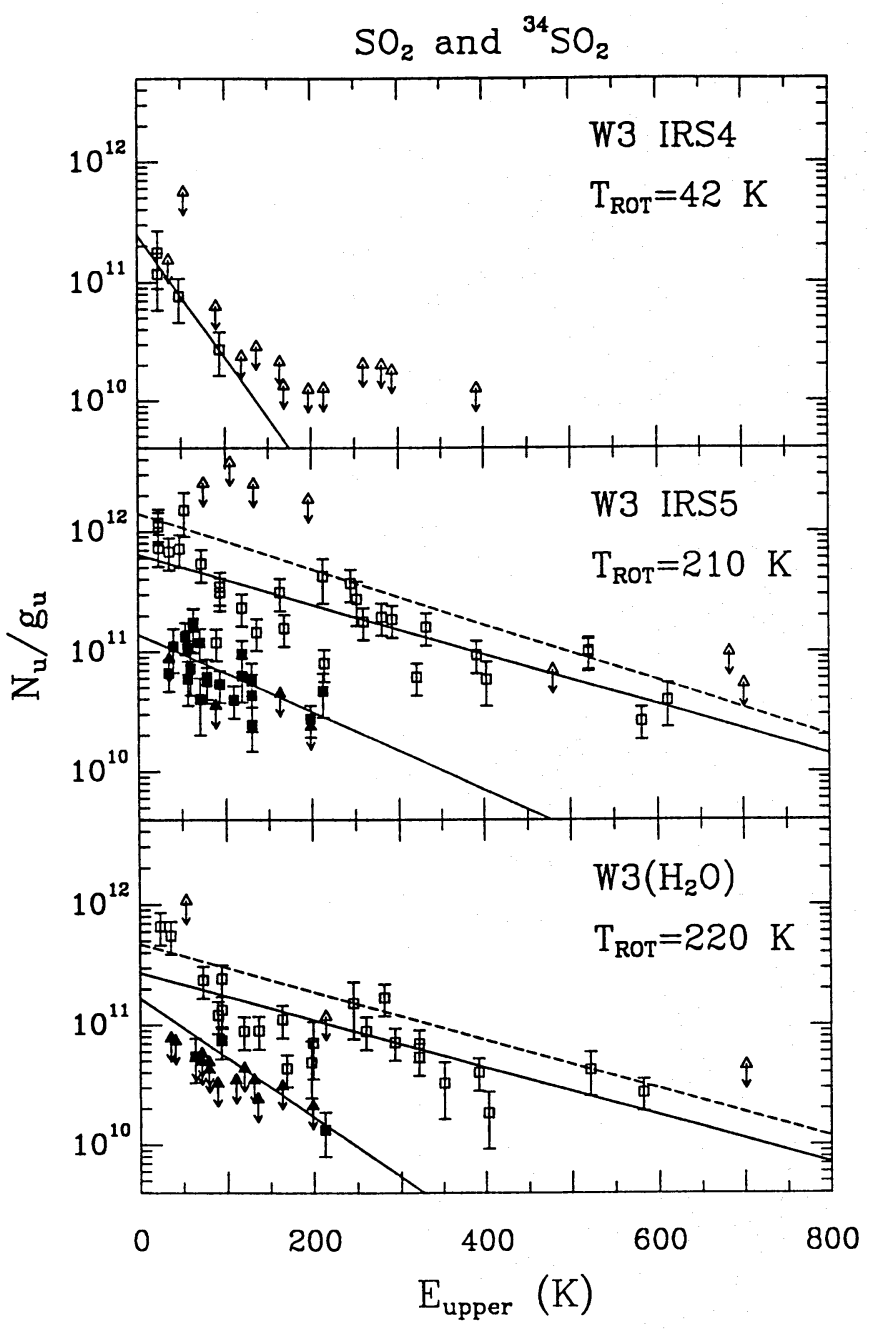

Fig. 3. Rotation diagrams for ${ }^{32} \mathrm{SO}_{2}$ (open symbols) and ${ }^{34} \mathrm{SO}_{2}$ (filled symbols) in the three sources. Squares indicate lines that have been detected; triangles denote upper limits. The full lines represent the least-squares fits using all of the detected transitions; the dashed lines indicate the fits through the ${ }^{32} \mathrm{SO}_{2} \Delta J=-1$ data only. The rotational temperatures shown refer to the least-squares fits to all the ${ }^{32} \mathrm{SO}_{2}$ data

The different beam sizes at 230 and $345 \mathrm{GHz}$ can also have some effect, but no systematic offset between the two data sets is found. Little direct information is available on the source sizes, but they may be smaller (of order a few ${ }^{\prime \prime}-10^{\prime \prime}$ ) than the JCMT beam. For source sizes as small as $2^{\prime \prime}$, the $230 \mathrm{GHz}$ data would be shifted by only 0.25 dex more than the $345 \mathrm{GHz}$ data, which is still insufficient to explain the scatter. Mitchell et al. (1990) have inferred from ${ }^{12} \mathrm{CO}$ and ${ }^{13} \mathrm{CO}$ infrared absorption lines towards IRS5 that the gas in front of the infrared source at $V_{\mathrm{LSR}} \approx-39 \mathrm{~km} \mathrm{~s}^{-1}$ consists of two components with comparable column densities: one with $T \approx 66 \mathrm{~K}$ and one with $T \approx 580 \mathrm{~K}$. We have attempted to fit such a two-component model to the IRS5 data, but did not obtain a significantly better result: most of the $\mathrm{SO}_{2}$ lines favor a temperature in between these two values. It is therefore more plausible that the scatter arises from optical depth effects. For $\mathrm{SO}_{2}$, the lines with
$\Delta J=J_{\mathrm{i}}-J_{\mathrm{f}}=0$ have intrinsically the largest line strengths $S$, followed by those with $\Delta J=1$ and -1 . It appears that the data for $\Delta J=0$ in IRS5 lie systematically lower than those for $\Delta J=-1$, implying that the former are saturated. This conclusion is strengthened by the fact that the isotope ratio of the column densities derived from the least-squares fits is ${ }^{32} \mathrm{SO}_{2} /{ }^{34} \mathrm{SO}_{2}=17 \pm 9$ for the $\Delta J=-1$ lines, but only $7 \pm 3$ for the $\Delta J=0$ lines. The cosmic ratio $\left[{ }^{32} \mathrm{~S}\right] /\left[{ }^{34} \mathrm{~S}\right]=22.59$, so that the $\Delta J=-1$ lines are nearly unsaturated. For $\mathrm{W} 3\left(\mathrm{H}_{2} \mathrm{O}\right)$, a similar trend is found, although many fewer ${ }^{34} \mathrm{SO}_{2}$ lines have been detected. The same conclusion was also reached by Sutton et al. (1991) to explain the scatter in their $\mathrm{SO}_{2}$ rotation diagrams towards Sgr B2(M).

Additional scatter can be caused by excitation effects: if the density is not high enough to thermalize the excitation or if a temperature gradient is present in the gas, the level populations cannot be characterized by a single excitation temperature. Since the lines with the largest line strengths also have the highest critical densities, they are more likely to be subthermally excited than those with lower line strengths. Finally, the populations of the higher energy levels may be affected by infrared pumping through the $\nu_{2}$ vibrational transition at $19 \mu \mathrm{m}$.

In Fig. 4, the rotation diagrams for the observed $\mathrm{CH}_{3} \mathrm{OH}$ lines in the three sources are presented. For IRS4, the data are again well fitted with a single, low excitation temperature $T_{\text {rot }} \approx 38 \pm 4 \mathrm{~K}$. The $\mathrm{CH}_{3} \mathrm{OH}$ lines towards IRS5 are too weak to provide a meaningful estimate of the rotational temperature. Towards W3 $\left(\mathrm{H}_{2} \mathrm{O}\right)$, the excitation is high, $T_{\text {rot }} \approx 200 \mathrm{~K}$, which is similar to the excitation temperature found for $\mathrm{NH}_{3}$ (Mauersberger et al. 1988). Again considerable scatter is seen in the data, but in this case, no systematic trend with line strength $S$ or transition type ( $A$ or $E$ ) could be found, so that the variations are most likely due to excitation effects. Part of the scatter may arise from the fact that some $\mathrm{CH}_{3} \mathrm{OH}$ emission is likely to arise from $\mathrm{W} 3(\mathrm{OH})$. If $\mathrm{CH}_{3} \mathrm{OH}$ has a similar excitation temperature as $\mathrm{NH}_{3}$ towards $\mathrm{W} 3(\mathrm{OH}), T_{\text {rot }} \approx 95 \mathrm{~K}$, only the lower-lying lines would be affected. However, we did not find evidence for such a second component at $-44 \mathrm{~km} \mathrm{~s}^{-1}$. Mauersberger et al. (1988) actually used a model with a power-law density and temperature variation towards $\mathrm{W} 3\left(\mathrm{H}_{2} \mathrm{O}\right)$ to fit the $\mathrm{NH}_{3}$ data, but we tend to follow Sutton et al. (1991), who explain the scatter in the rotation diagram for the Sgr B2(M) methanol lines by non-LTE effects in the excitation. The best single temperature fits to the data are summarized in Table 2.

\subsection{Excitation of $\mathrm{H}_{2} \mathrm{CO}$}

Figure 5 contains the rotation diagrams for $\mathrm{H}_{2} \mathrm{CO}$. In general, the $\mathrm{H}_{2} \mathrm{CO}$ data follow the same trend as found for the other two molecules, in the sense that the excitation temperature is around $50 \mathrm{~K}$ for IRS4, somewhat higher towards IRS5 and highest towards $\mathrm{W} 3\left(\mathrm{H}_{2} \mathrm{O}\right)$. However, the figure also shows that for IRS5 and IRS4, the $\mathrm{H}_{2} \mathrm{CO}$ data observed at $230 \mathrm{GHz}$ lie systematically higher than those at $345 \mathrm{GHz}$. The difference of nearly an order of magnitude is too large to be due to different beam size effects or calibration uncertainties. Also, the observed upper 


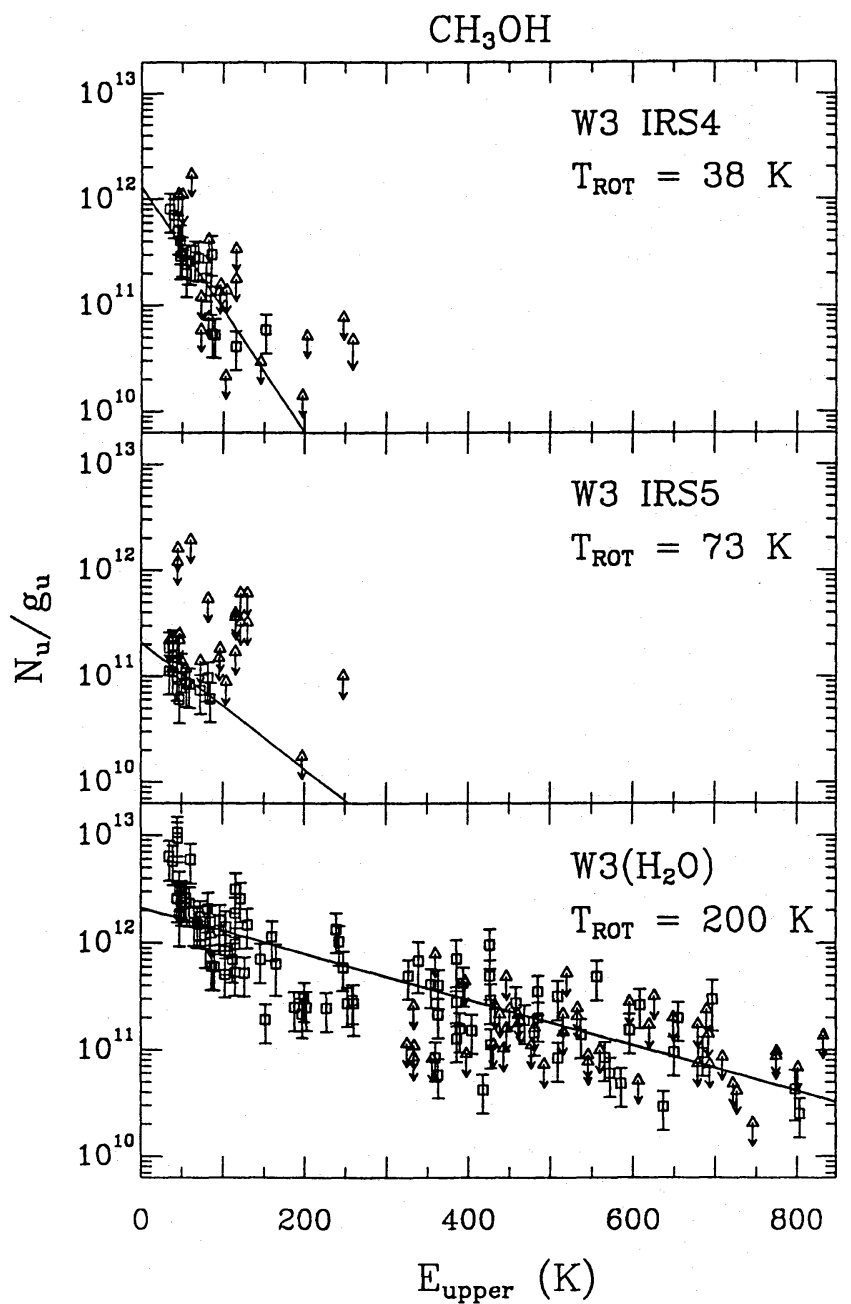

Fig. 4. $\mathrm{CH}_{3} \mathrm{OH}$ rotation diagrams for the three sources. Squares indicate lines that have been detected; triangles denote upper limits. The full lines represent the least-squares fits using all of the detected transitions. The corresponding rotational temperatures are indicated

Table 2. Excitation temperatures and column densities

\begin{tabular}{llccc}
\hline Source & Molecule & $\begin{array}{c}T_{\text {rot }} \\
(\mathrm{K})\end{array}$ & $\begin{array}{c}N \\
\left(10^{13} \mathrm{~cm}^{-2}\right)\end{array}$ & $\begin{array}{c}\mathrm{X}^{a} \\
10^{-10}\end{array}$ \\
\hline W3 IRS4 & $\mathrm{H}_{2} \mathrm{CO}$ & $50:^{b}$ & $8 \pm 2^{c}$ & $2.9 \pm 1.1^{c}$ \\
& $\mathrm{CH}_{3} \mathrm{OH}$ & $38 \pm 4$ & $37 \pm 11$ & $13 \pm 5.5$ \\
& $\mathrm{SO}_{2}$ & $42 \pm 13$ & $7.7 \pm 5.1$ & $2.8 \pm 2.0$ \\
W3 IRS5 & $\mathrm{H}_{2} \mathrm{CO}$ & $64:^{b}$ & $8 \pm 2^{c}$ & $1.8 \pm 0.7^{c}$ \\
& $\mathrm{CH}_{3} \mathrm{OH}$ & $73 \pm 32^{b}$ & $16 \pm 12$ & $3.5 \pm 2.8$ \\
& $\mathrm{SO}_{2}$ & $187 \pm 29$ & $410 \pm 162$ & $90 \pm 45$ \\
& ${ }^{34} \mathrm{SO}_{2}$ & $134 \pm 30$ & $25 \pm 9$ & $5.5 \pm 2.6$ \\
W3( $\left.\mathrm{H}_{2} \mathrm{O}\right)$ & $\mathrm{H}_{2} \mathrm{CO}$ & $149:^{b}$ & $40 \pm 10^{c}$ & $11 \pm 4.3^{c}$ \\
& $\mathrm{CH}_{3} \mathrm{OH}$ & $203 \pm 8$ & $754 \pm 68$ & $211 \pm 66$ \\
& $\mathrm{SO}_{2}$ & $216 \pm 108$ & $170 \pm 215$ & $47 \pm 20$ \\
& ${ }^{34} \mathrm{SO}_{2}$ & $88 \pm 27$ & $15 \pm 10$ & $4.2 \pm 3.1$ \\
\hline
\end{tabular}

${ }^{a}$ The abundances $X$ are obtained using the $\mathrm{H}_{2}$ column densities derived from $\mathrm{C}^{17} \mathrm{O} 3-2$ observations (see Table 3). ${ }^{b} T_{\text {rot }}$ not well determined, see text. ${ }^{c}$ As derived from statistical equilibrium calculations.

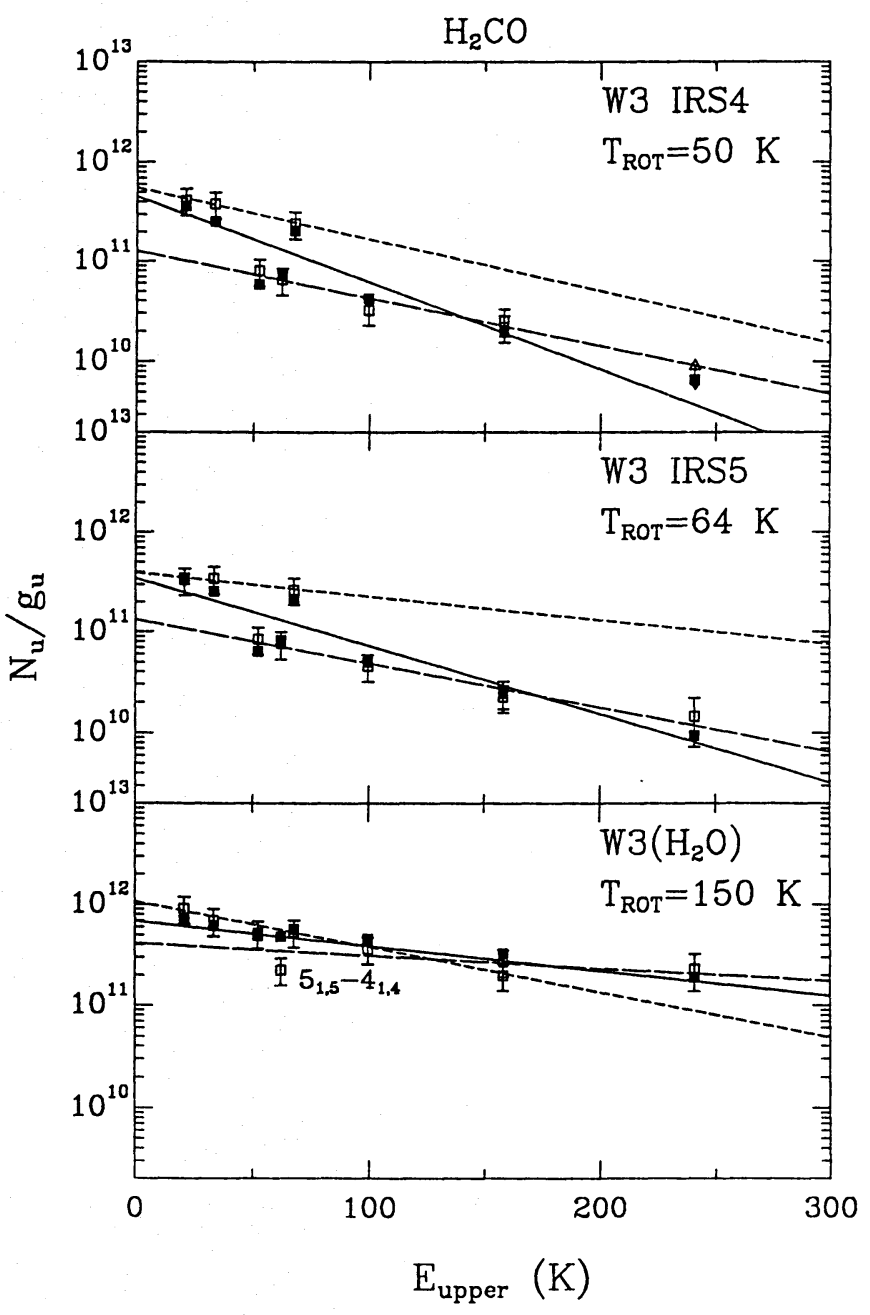

Fig. 5. $\mathrm{H}_{2} \mathrm{CO}$ rotation diagram for the three sources. The open symbols represent the measurements, the solid symbols the statistical equilibrium calculations. Squares indicate lines that have been detected; triangles denote upper limits. The full lines represent the least-squares fit through all the detected transitions. The short-dashed lines represent the fit through the $230 \mathrm{GHz}$ data; the long-dashed lines through the $345 \mathrm{GHz}$ data. The separation between these two dashed lines is a measure of the total number density $n\left(\mathrm{H}_{2}\right)$ (see text). The rotational temperatures shown correspond to the least-squares fits through all the data

limits for $\mathrm{H}_{2}^{13} \mathrm{CO}$ indicate that the lines cannot be very optically thick in these two sources. In order to investigate the excitation of $\mathrm{H}_{2} \mathrm{CO}$ in more detail, we performed statistical equilibrium calculations for the molecule in the same way as described by Jansen et al. (1993) and van Dishoeck et al. (1993b). Because $\mathrm{H}_{2} \mathrm{CO}$ is a near-prolate rotor, transitions between levels in different $K_{P}$ ladders are radiatively forbidden. As a result, ratios of lines originating from different $K_{P}$ ladders generally are excellent indicators of the kinetic temperature of the gas, whereas ratios of lines within one ladder provide good density diagnostics (see also Mangum \& Wootten 1993). Examples of the sensitivity of the line ratios to the physical conditions are illustrated in Fig. 6. If the observed line ratios in W3 are plotted 
in this figure, we find $T=55_{-10}^{+20}, 100_{-20}^{+40}$ and $220 \pm 40 \mathrm{~K}$ for IRS4, IRS5 and W3 $\left(\mathrm{H}_{2} \mathrm{O}\right)$, respectively, with $\mathrm{H}_{2}$ densities of $1 \cdot 10^{6}, 1 \cdot 10^{6}$ and $3 \cdot 10^{6} \mathrm{~cm}^{-3}$. Mangum \& Wootten find $T=75 \mathrm{~K}$ for IRS4, comparable to our estimate, and $110 \mathrm{~K}$ for the $\mathrm{W} 3\left(\mathrm{H}_{2} \mathrm{O}\right) / \mathrm{W} 3(\mathrm{OH})$ region, which is much lower than our value. This difference can be due to the fact that their position is slightly offset from ours and their beam bigger, thus sampling more of the $\sim 100 \mathrm{~K}$ gas surrounding $\mathrm{W} 3(\mathrm{OH})$. Their inferred densities towards IRS4 of $2.5 \cdot 10^{5} \mathrm{~cm}^{-3}$ and towards $\mathrm{W} 3\left(\mathrm{H}_{2} \mathrm{O}\right) / \mathrm{W} 3(\mathrm{OH})$ of $8.9 \cdot 10^{5} \mathrm{~cm}^{-3}$ are also much lower than our values. For both sources this seems to be caused primarily by the observed strength of the $5_{0,5}-4_{0,4}$ line: our value is almost twice as high as theirs, enhancing the density by a factor of 3-4. The fact that our density value is derived from a more extended data set with a greater range of transitions leads us to favor the higher densities. The best-fitting column densities are approximately $8 \cdot 10^{13}, 8 \cdot 10^{13}$ and $4 \cdot 10^{14} \mathrm{~cm}^{-2}$ for IRS4, IRS5 and W3 $\left(\mathrm{H}_{2} \mathrm{O}\right)$, respectively, and for all three sources $N_{\text {ortho }} / N_{\text {para }}$ is close to three.

The resulting model intensities for the best-fitting parameters have been included in the rotation diagrams shown in Fig. 5, and it is seen that a nearly perfect match with the observations is obtained. The model results show that the rotation diagram only gives a single line for densities $n>$ few $\cdot 10^{6} \mathrm{~cm}^{-3}$. Thus, the densities in IRS5 and IRS4 have to be lower than this value to show the "split" between the $230(J=3-2)$ and $345 \mathrm{GHz}$ $(J=5-4)$ data. In $\mathrm{W} 3\left(\mathrm{H}_{2} \mathrm{O}\right)$ the density must be closer to $10^{7} \mathrm{~cm}^{-3}$, since the data points for this source deviate much less from a single line. The prominent exception is the $5_{1,5}-4_{1,4}$ line at $E_{\mathrm{u}}=62.45 \mathrm{~K}$, which has a large line strength $\left(\mu^{2} S=78\right)$. Although the statistical equilibrium calculations do not give a high optical depth $(\tau<1)$, the line could still be optically thick if the source size is smaller than the beam. Clues to the amount of saturation are normally obtained by comparison with an isotope, but unfortunately the $\mathrm{H}_{2}^{13} \mathrm{CO}_{1,5}-4_{1,4}$ line in this source is blended with $\mathrm{H}_{2} \mathrm{CS} 10_{2,9}-9_{2,8}$. The $\mathrm{H}_{2} \mathrm{CO} / \mathrm{H}_{2}^{13} \mathrm{CO}$ ratio found for other lines is $\sim 17$ for $3_{1,2}-2_{1,1}\left(\mu^{2} S=43\right)$ and $\sim 45$ for $5_{0,5}-4_{0,4}\left(\mu^{2} S=27\right)$. Since both transitions have lower line strengths, they suggest that some saturation is likely for the $5_{1,5}-4_{1,4}$ line.

\subsection{Total column density and abundances}

From the rotation diagrams presented in Figs. 3 and 4, the column densities of $\mathrm{SO}_{2}$ and $\mathrm{CH}_{3} \mathrm{OH}$ in the three sources can be derived. For $\mathrm{SO}_{2}$, we only used the fit to the weaker $\Delta J=-1$ lines to determine its column density in IRS5 and $\mathrm{W} 3\left(\mathrm{H}_{2} \mathrm{O}\right)$. These values agree well with those derived from the ${ }^{34} \mathrm{SO}_{2}$ lines assuming a normal isotope ratio. The results of the least-squares fits are summarized in Table 2. The $\mathrm{H}_{2} \mathrm{CO}$ column densities were constrained from the model statistical equilibrium calculations.

In order to determine the molecular abundances, the total $\mathrm{H}_{2}$ column densities along the lines of sight need to be known. These can be constrained from isotopic CO observations, or from millimeter continuum data. As discussed by Phillips et al. (1992), $\mathrm{C}^{18} \mathrm{O} 2-1$ observations suggest similar column densi- ties towards the three sources of about $2 \cdot 10^{23} \mathrm{~cm}^{-2}$. Comparable values are obtained from dust mass estimates and from the infrared absorption lines (Richardson et al. 1989; Mitchell et al. 1990). The $\mathrm{C}^{17} \mathrm{O} 3-2$ observations obtained in this work, however, show that even the $\mathrm{C}^{18} \mathrm{O}$ lines may still be somewhat optically thick. The data are presented in Table 3 . The beamaveraged $\mathrm{C}^{17} \mathrm{O}$ column densities have been derived using the temperatures and densities derived from the $\mathrm{H}_{2} \mathrm{CO}$ lines in the previous section, except for $\mathrm{W} 3\left(\mathrm{H}_{2} \mathrm{O}\right)$ where a slightly lower CO column-averaged temperature, $T \approx 100 \mathrm{~K}$, was adopted. These values are not sensitive to an order of magnitude change in density, but scale directly with adopted temperature. The resulting beam-averaged $\mathrm{H}_{2}$ column densities were obtained assuming that the ${ }^{12} \mathrm{C}^{16} \mathrm{O} /{ }^{12} \mathrm{C}^{17} \mathrm{O}$ ratio is equal to the ratio $\left[{ }^{16} \mathrm{O}\right] /\left[{ }^{17} \mathrm{O}\right]=2.6 \cdot 10^{3}$ (Barnes et al. 1991) and that the gasphase $\mathrm{CO}$ abundance $\mathrm{CO} / \mathrm{H}_{2}=8 \cdot 10^{-5}$. This latter value has been determined explicitly for W3 IRS5 by Tielens et al. (1991) from observations of the $\mathrm{CO}$ infrared absorption lines combined with the measured silicate optical depth, assuming a standard gas/dust ratio. The amount of solid CO towards IRS5 is found to be less than one percent of that of gas-phase $\mathrm{CO}$. We assume that the same values apply to IRS4 and $\mathrm{W} 3\left(\mathrm{H}_{2} \mathrm{O}\right)$ and that $\mathrm{CO}$ is also not much depleted in these high temperature regions.

The resulting beam-averaged abundances $X=N(\mathrm{X}) / N\left(\mathrm{H}_{2}\right)$ are included in Table 2. It is seen that $\mathrm{SO}_{2}$ is nearly two orders of magnitude more abundant towards IRS5 than towards IRS4, whereas $\mathrm{CH}_{3} \mathrm{OH}$ is more than an order of magnitude more abundant towards $\mathrm{W} 3\left(\mathrm{H}_{2} \mathrm{O}\right)$ than towards the other two sources. Since the source size of $\mathrm{CH}_{3} \mathrm{OH}$ in $\mathrm{W} 3\left(\mathrm{H}_{2} \mathrm{O}\right)$ is likely to be smaller than that of $\mathrm{C}^{17} \mathrm{O}$ (Wilson et al. 1991; Turner \& Welch 1984), the effect may be even larger.

\section{Discussion}

The previous sections have shown that there appear to be genuine variations in physical parameters and chemical abundances between the three positions. To what extent are they related to the evolutionary state of the object? An equally important question is the inverse one: given these variations, can we identify molecules that are uniquely associated with a particular phase in the evolution (cf. van Dishoeck et al. 1993a)? In the current favorite scenario (Blake et al. 1987; Williams \& Hartquist 1991), the chemistry in the quiescent cloud prior to star formation is dominated by low-temperature gas-phase ion-molecule reactions. During the subsequent collapse phase, the densities increase to such high values that most molecules condense onto the grains. Here the chemical composition may be modified by grain-surface reactions. After the star has formed, its radiation will heat the gas and the grains, so that volatile molecules are returned to the gas phase, presumably in a sequence according to their condensation temperatures. The high temperatures and radiation from the star can further affect the grain chemistry by creating more complex species (d'Hendecourt et al. 1985). Subsequent gas-phase reactions can modify the chemical abundances as well, but eventually, after about $10^{5} \mathrm{yr}$, the abundances 

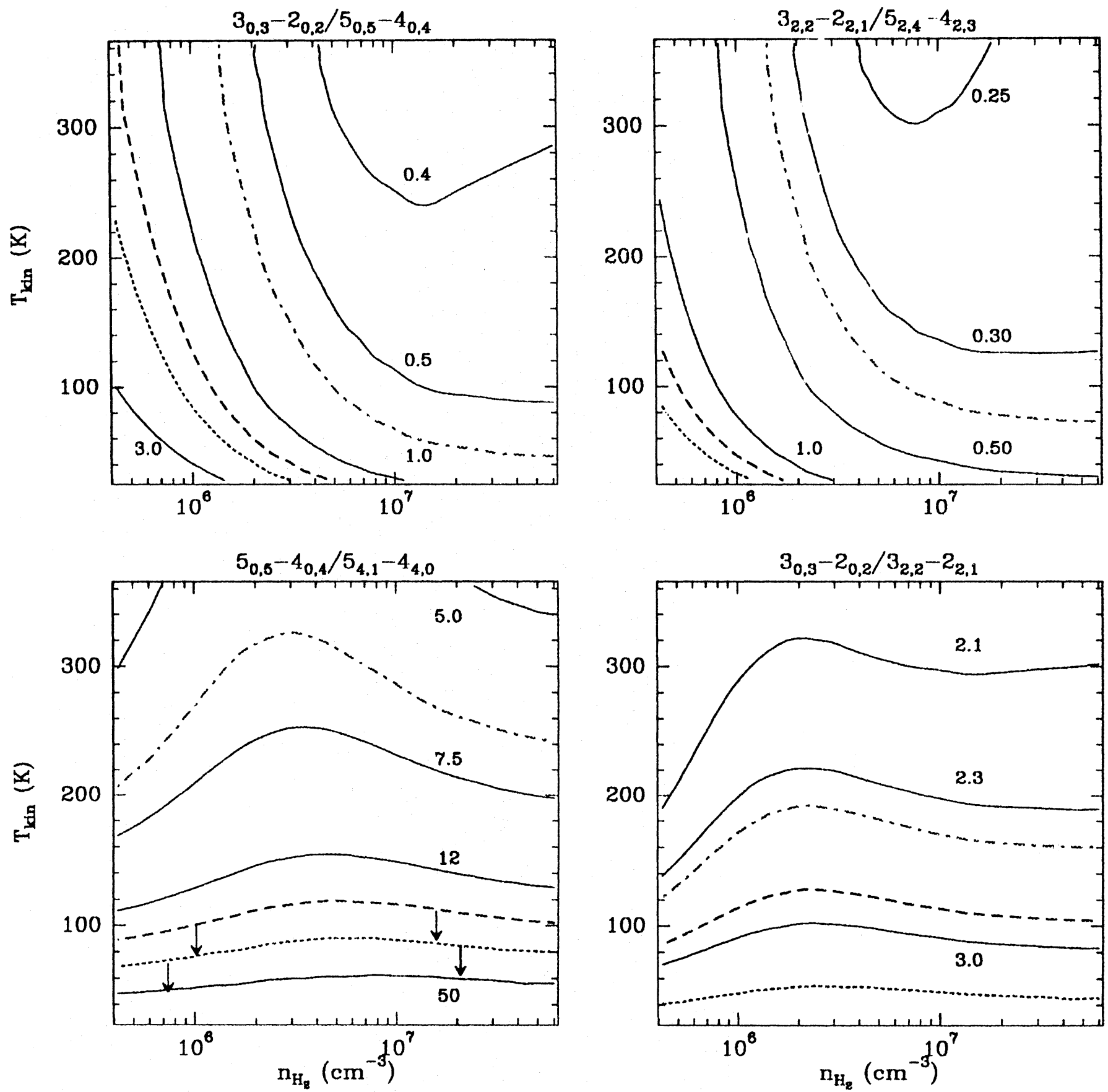

Fig. 6. $\mathrm{H}_{2} \mathrm{CO}$ line ratios as functions of density and temperature obtained from statistical equilibrium calculations. The full lines indicate the model results. The adopted column density is $N\left(\mathrm{H}_{2} \mathrm{CO}\right)=8 \times 10^{13} \mathrm{~cm}^{-2}$ and $\Delta V=5 \mathrm{~km} \mathrm{~s}^{-1}$, but the model line ratios are not sensitive to small variations in these parameters. The dashed lines show the observed values for IRS4 (short dash), IRS5 (long dash) and W3( $\left.\mathrm{H}_{2} \mathrm{O}\right)$ (dot-dash)

will be reset to the quiescent dense cloud values (Charnley et al.1992; Caselli et al. 1993).

Of the three regions W3 IRS4 appears to be the most evolved one. Star formation has obviously taken place in this region, but the chemical composition resembles that of a quiescent cloud such as the Orion ridge, in which only simple molecules are found. The radio continuum map of Colley (1980) of this region (W3C) has the same morphology as the blister-like $\mathrm{H}$ II regions described by Icke et al. (1980). The star apparently formed near the far side of the cloud, where its ultraviolet photons created an ionized region. The ionization front continued to expand until it encountered the outer edge of the cloud. Because of the much lower pressure, the ionized gas dispersed rapidly on that side, like the burst of a blister. On the other side of the $\mathrm{H}$ II region, the ionization front is still expanding further into the molecular gas, but much more slowly, because of the higher density. After the 
Table 3. $\mathrm{C}^{17} \mathrm{O}$ model parameters

\begin{tabular}{lcccccc}
\hline Source & $\begin{array}{c}\int T_{M B}(3-2) d V \\
\left(\mathrm{~K} \mathrm{~km} \mathrm{~s}^{-1}\right)\end{array}$ & $\begin{array}{c}\Delta V \\
\left(\mathrm{~km} \mathrm{~s}^{-1}\right)\end{array}$ & $\begin{array}{c}T^{a} \\
(\mathrm{~K})\end{array}$ & $\begin{array}{c}n^{a} \\
\left(10^{6} \mathrm{~cm}^{-3}\right)\end{array}$ & $\begin{array}{c}N\left(\mathrm{C}^{17} \mathrm{O}\right) \\
\left(10^{16} \mathrm{~cm}^{-2}\right)\end{array}$ & $\begin{array}{c}N\left(\mathrm{H}_{2}\right) \\
\left(10^{23} \mathrm{~cm}^{-2}\right)\end{array}$ \\
\hline W3 IRS4 & 16.2 & 3.5 & 55 & 1.0 & 0.87 & $3 \pm 1$ \\
W3 IRS5 & 22.0 & 4.1 & 100 & 1.0 & 1.4 & $5 \pm 1$ \\
W3( $\left.\mathrm{H}_{2} \mathrm{O}\right)$ & 16.7 & 4.4 & 100 & 3.0 & 1.1 & $4 \pm 1$ \\
\hline
\end{tabular}

${ }^{a}$ Temperatures and densities as derived from $\mathrm{H}_{2} \mathrm{CO}$ observations, except for $\mathrm{W} 3\left(\mathrm{H}_{2} \mathrm{O}\right)$ where

$T=100 \mathrm{~K}$ is assumed.

blister has opened, the high internal pressure of the early times no longer exists, leading to lower temperatures and densities, and removing the conditions for $\mathrm{H}_{2} \mathrm{O}$ masers. Indeed, no $\mathrm{H}_{2} \mathrm{O}$ masers are found near IRS4. In this stage, the source probably does not possess outflows anymore, but the complex velocity field around IRS4 (Hayashi et al. 1989; Mitchell et al. 1992) prevent a definite statement from being made. The blister scenario can account in a natural way for the small displacement of $7^{\prime \prime}$ seen between the IR peak (Wynn-Williams et al. 1972) and the radio-continuum shell (Colley 1980). Using an ionization front velocity of $1 \mathrm{~km} \mathrm{~s}^{-1}$ and an extent of $15^{\prime \prime}$ (Colley 1980), the time since the start of the expansion of the ionization front must be about $10^{5} \mathrm{yr}$. Not only is this enough time for the $\mathrm{HII}$ region to evolve to the radio structure seen now, but it is also sufficient time for the abundances to return to the quiescent cloud values. If gas-phase ion-molecule chemistry is indeed dominant again at this stage, it would account for the less complex chemistry observed at this position and would be consistent with the smaller line widths.

In contrast, $\mathrm{W} 3\left(\mathrm{H}_{2} \mathrm{O}\right)$ appears to be more deeply embedded and younger than W3 IRS4. It shows only a weak, barely resolved $\left(<1^{\prime \prime}\right)$ radio continuum (Guilloteau et al. 1985), and the visual extinction towards the source is about 1000 magnitudes (Thronson \& Harper 1979; Turner \& Welch 1984). The radiation pressure from the young star has created a surrounding warm, dense shell of gas and dust with conditions favorable for $\mathrm{H}_{2} \mathrm{O}$ maser emission and for creating more complex organic molecules. The richness in molecular lines, as visible in Figs. 1 and 2 , imply a remarkable chemical evolution compared with the surrounding quiescent cloud. This suggests the presence of an object like the Orion hot core, in which processed molecules such as $\mathrm{CH}_{3} \mathrm{OH}$ have evaporated from the grains (Blake et al. 1987; Charnley et al. 1992; Caselli et al. 1993). The detection of HDO towards W3 $\left(\mathrm{H}_{2} \mathrm{O}\right)$ by Jacq et al. (1990) also supports this picture. The age of $\mathrm{W} 3\left(\mathrm{H}_{2} \mathrm{O}\right)$ is less than $10^{5} \mathrm{yr}$, and is probably of the order of several times $10^{4} \mathrm{yr}$. The absence of any detailed information concerning an outflow makes it difficult to estimate a dynamical age. The only hint at the presence of an outflow is given by Mauersberger et al. (1988), who deduce from the continuum emission found by Guilloteau et al. (1985) that an outflow has blown a hole in the surrounding molecular medium through which the ionized gas escapes, as in the BN-object.
IRS5 is most likely the youngest object of the three, and has only a very weak, unresolved $\left(<1^{\prime \prime}\right)$ radio continuum source associated with it (Colley 1980), implying that the embedded object has only recently become hot enough to produce ionizing photons. However, the source has already developed a powerful outflow with an estimated dynamical age of about (1-3) $10^{4}$ yr (Mitchell et al. 1991, 1992). From interferometer measurements Claussen et al. (1984) deduce a dynamical age of about 1600 years, but this refers to a more compact structure than the extended outflow seen by Mitchell et al. In either case, the dynamical age is young. The total column density along the line of sight is high, but the abundances of volatile molecules like formaldehyde and methanol are low. Surprisingly, the sulfur dioxide abundance is high in comparison with most sources. This high $\mathrm{SO}_{2}$ abundance is reminiscent of that found in the Orion plateau, and probably results from shock chemistry and shock destruction of refractory grain cores in a high density environment. The shocks most likely arise when the strong outflow from IRS5 impacts the surrounding material, although other energetic events could play a rôle as well. Interestingly, the rotational temperature of $\mathrm{SO}_{2}$ is similar to the temperature of 200-270 K found by Mitchell et al. (1991) for the blue-shifted $\mathrm{CO}$ infrared absorption. Mitchell et al. deduce that $N(\mathrm{CO})$ is about $5 \cdot 10^{17} \mathrm{~cm}^{-2}$ in the outflow, compared with his total $\mathrm{CO}$ column density of $2.4 \cdot 10^{19} \mathrm{~cm}^{-2}$. If we assume that the sulfur dioxide originates only in the outflow and that about $2 \%$ of all the gas is in the outflow, the sulfur dioxide abundance increases from $9 \cdot 10^{-9}$ to about $4.5 \cdot 10^{-7}$, which is very close to the Orion plateau value of $5.2 \cdot 10^{-7}$ (Blake et al. 1987).

The $\mathrm{SO}_{2}$ chemistry is not yet well understood, but may be a very sensitive indicator of evolutionary stage. $\mathrm{SO}_{2}$ is also seen towards W3 $\left(\mathrm{H}_{2} \mathrm{O}\right)$, but not towards the more evolved W3 IRS4. A similar picture is found in other sources: $\mathrm{SO}_{2}$ is very prominent in Orion $/ \mathrm{KL}$, but its abundance is very low in the young stellar object Orion 1.'5S, which has a comparable luminosity (McMullin et al. 1993). Presumably the Orion S source is even younger than W3 IRS5, as evidenced by its highly collimated outflow (Schmid-Burgk et al. 1990). The $\mathrm{SO}_{2}$ chemistry does not seem to depend much on luminosity or mass of the young stellar object, since lines of $\mathrm{SO}_{2}$ also appear strongly in spectra towards the low-mass star-forming region IRAS $16293-2422$ (Blake et al. 1993). 
The methanol abundance towards IRS5 is remarkably low, even compared with dark cloud values (cf. Van Dishoeck et al. 1993a; Ohishi et al. 1992), suggesting that most of the molecules, outside the outflow(s), are still frozen onto the grains. This is consistent with the fact that Allamandola et al. (1992) find from near infrared absorption observations a solid methanol column density of more than about $10^{18} \mathrm{~cm}^{-2}$ towards IRS5, i.e. more than four orders of magnitude larger than found in the gas phase. Eventually the processed molecules will evaporate from the grains when the temperature has risen to about $100 \mathrm{~K}$ (Mumma et al. 1993). The fact that the gas temperature derived from the $\mathrm{H}_{2} \mathrm{CO}$ observations is close to $100 \mathrm{~K}$ and that the dust temperature is about $65 \mathrm{~K}$ (Gordon 1987) suggests that this process is just starting in the central part of IRS5. It is possible that we are witnessing at IRS5 the birth of a hot core-like object.

\section{Conclusions}

Submillimeter observations of three sources in the W3 molecular cloud are presented, together with an analysis of the lines of three molecules, $\mathrm{CH}_{3} \mathrm{OH}, \mathrm{H}_{2} \mathrm{CO}$ and $\mathrm{SO}_{2}$. These molecules are particularly valuable probes of the physical conditions, such as temperature and density, and also have very different chemical characteristics. The kinetic temperature inferred from the $\mathrm{H}_{2} \mathrm{CO}$ data is lowest at the IRS4 position ( $T \approx 55 \mathrm{~K}$ ), increasing to $\sim 100 \mathrm{~K}$ at the IRS5 position and $\sim 200 \mathrm{~K}$ at the $\mathrm{W} 3\left(\mathrm{H}_{2} \mathrm{O}\right)$ source. The density is about $10^{6} \mathrm{~cm}^{-3}$ at IRS4 and IRS5, and a few $\cdot 10^{6} \mathrm{~cm}^{-3}$ at $\mathrm{W} 3\left(\mathrm{H}_{2} \mathrm{O}\right)$.

The three sources not only have different physical conditions, but also show strong chemical variations. The methanol abundance towards IRS5 is more than an order of magnitude lower than towards $\mathrm{W} 3\left(\mathrm{H}_{2} \mathrm{O}\right)$. This extremely low gasphase methanol abundance in IRS5 compared with the observed amount of solid-state methanol is very strong evidence for freezing out of molecules onto the grains. The high gas and dust temperature towards IRS5 suggest that these molecules will shortly evaporate off the grains, at which stage the source will probably start to resemble a hot-core type region.

In contrast, the $\mathrm{SO}_{2}$ abundance is very high towards IRS5, moderate towards $\mathrm{W} 3\left(\mathrm{H}_{2} \mathrm{O}\right)$, but low towards IRS4. Towards IRS5, the $\mathrm{SO}_{2}$ rotational temperature is high, and is similar to that found by Mitchell et al. (1991) for CO in the outflow. This fact, together with the similarity in abundances found in Orion/KL (Blake et al. 1987), strongly suggests that $\mathrm{SO}_{2}$ originates in the region where the outflow runs into the ambient material. The limited data on $\mathrm{SO}_{2}$ further suggest that this molecule is a very sensitive indicator of evolutionary stage, and that its abundance probably peaks at an earlier stage than that of the complex organic molecules.

Acknowledgements. The authors are indebted to Fred Baas and the JCMT staff for assistance with the observing runs, and to Peter Schilke and Eric Herbst for help with updating the $\mathrm{CH}_{3} \mathrm{OH}$ line catalogs. They thank Geoff Blake, Graeme Oldham and Ken Richardson for useful discussions. Frank Israel provided the blister scenario for IRS4. This research was supported by the Netherlands Organization for Scientific Research, NWO, and by NSF contract AST 90-15755 to the California Institute of Technology.

\section{References}

Allamandola, L.J., Sandford, S.A., Tielens, A.G.G.M., Herbst, T.M. 1992, ApJ 399, 134.

Baas, F. 1992, private communication.

Barnes, I.L., Chang, T.L., De Bièvre, P. et al., 1991 Pure \& Appl. Chem. 63, 991.

Blake, G.A., Sutton, E.C., Masson, C.R., Phillips, T.G. 1987, ApJ 315,621 .

Blake, G.A., Van Dishoeck, E.F., Jansen, D.J., Groesbeck, T.D. 1993, ApJ, in press.

Caselli, P., Hasegawa, T.I., Herbst, E. 1993, ApJ 408, 548.

Charnley, S.B., Tielens, A.G.G.M., Millar, T.J. 1992, ApJ 399, L71.

Claussen, M.J., Berge, G.L., Heiligman, G.M. et al., 1984, ApJ 285, L79.

Colley, D. 1980, MNRAS 193, 495.

D’Hendecourt, L.B., Allamandola, L.J., Greenberg, J.M. 1985, A\&A $152,130$.

Dickel, H.R., Dickel, J.R., Wilson, W.J., Werner, M.W. 1980, ApJ 237, 711 .

Georgelin, Y.M., Georgelin, Y.P. 1976, A\&A 49, 57.

Gordon, M.A. 1987, ApJ 316, 258.

Guilloteau, S., Baudry, A., Walmsley, C.M. 1985, A\&A 153, 179.

Hayashi, M., Kobayashi, H., Hasegawa, T. 1989, ApJ 340, 298.

Icke, V., Gatley, I., Israel, F.P. 1980, ApJ 236, 808.

Jacq, T., Walmsley, C.M., Henkel, C. et al., 1990, A\&A 228, 447.

Jansen, D.J., Van Dishoeck, E.F., Black, J.H., 1993, A\&A, in press.

Kutner, M.L., Ulich, B.L. 1981, ApJ 250, 341.

Mangum, J.E., Wootten, A. 1993, ApJS, in press.

Matthews, H.E. 1991, in The James Clerk Maxwell Telescope: A guide for the prospective user.

Mauersberger, R., Wilson, T.L., Henkel, C. 1988, A\&A 201, 123.

McMullin, J.P., Mundy, L.G., Blake, G.A. 1993, ApJ. 405, 599.

Mitchell, G.F., Maillard, J.-P., Allen, M. et al., 1990, ApJ 363, 554.

Mitchell, G.F., Maillard, J.-P., Hasegawa, T.I. 1991, ApJ 371, 342.

Mitchell, G.F., Hasegawa, T.I., Schella, J. 1992, ApJ 386, 604.

Mumma, M.J., Weissman, P.R., Stern, S.A. 1993, in Protostars and Planets III, eds. E.H. Levy and J.I. Lunine (University of Arizona Press, Tucson), p. 1177.

Ohishi, M., Irvine, W.M., Kaifu, N. 1992, in The Astrochemistry of Cosmic Phenomena, IAU Symposium 150, ed P.D. Singh (Kluwer, Dordrecht), p. 171.

Phillips, T.G., Van Dishoeck, E.F., Keene, J. 1992, ApJ 399, 533.

Richardson, K.J., Sandell, G., White, G.J. et al., 1989, A\&A 221, 95.

Schmid-Burgk, J. Güsten, R. Mauersberger, R. et al., 1990, ApJ 362, L25.

Sutton, E.C., Jaminet, P.A., Danchi, W.C., Blake, G.A. 1991, ApJS $77,255$.

Thronson Jr., H.A., Harper, D.A. 1979, ApJ 230, 133.

Tielens, A.G.G.M., Tokunaga, A.T., Geballe, T.R., Baas. F. 1991, ApJ 381, 181.

Turner, J.L., Welch, W.J. 1984, ApJ 287, L81.

Van Dishoeck, E.F., Blake, G.A., Draine, B.T., Lunine, J.I. 1993a, in Protostars and Planets III, eds. E.H. Levy and J.I. Lunine (University of Arizona Press, Tucson), p. 163.

Van Dishoeck, E.F., Jansen, D.J., Phillips, T.G. 1993b, A\&A, in press. Williams, D.A., Hartquist, T.W. 1991, MNRAS 251, 351.

Wilson, T.L., Johnston, K.J., Mauersberger, R. 1991, A\&A 251, 220.

Wynn-Williams, C.G., Becklin, E.E., Neugebauer, G. 1972, MNRAS $160,1$.

This article was processed by the author using Springer-Verlag $\operatorname{LAT}_{\mathrm{E}} \mathrm{X}$ A\&A style file version 3 . 